\title{
A green production strategies for carbon-sensitive products with a carbon cap policy
}

\author{
Ma, C. ${ }^{a}$, Liu, X..$^{b,}{ }^{,}$, Zhang, H. $^{b}$, Wu, Y. ${ }^{b}$ \\ ${ }^{a}$ Tian Fu College of SouthWestern University of Finance and Economics, Chengdu, China \\ bInternational Business School, Sichuan Technology and Business University, Chengdu, China
}

\begin{abstract}
A B S T R A C T
This paper discusses the production strategies used by manufacturers of carbon-sensitive products that have a carbon cap policy under both deterministic demand and stochastic demand. In this study, we examine green manufacturing strategies for carbon-sensitive products under carbon cap policy regulations. We primarily consider the two scenarios of deterministic demand and stochastic demand. When the carbon cap policy regulation has no restriction to the production of the manufacturers, the higher the carbon sensitivity coefficient of the product, the lower the profit of the manufacturing enterprise. When carbon cap policy regulation of manufacturing enterprise production is a constraint, for the deterministic demand, with the higher carbon sensitive coefficient, manufacturing enterprise profit is higher; for stochastic demand, with the increasingly high carbon sensitive coefficient, manufacturing enterprise profit is low. Through the above research, the conclusion of this paper has reference value and guiding role to carbon-sensitive products' green production strategies with a carbon cap policy.
\end{abstract}

\section{ARTICLE INFO}

Keywords:

Production strategy

Carbon sensitive

Carbon cap policy

*Corresponding author:

uestc-vip@163.com

(Liu, X.)

Article history:

Received 12 June 2016

Revised 5 July 2016

Accepted 18 July 2016

\section{References}

[1] Hong, Z., Chu, C., Yu, Y. (2012). Optimization of production planning for green manufacturing, In: 9th IEEE International Conference on Networking, Sensing and Control (ICNSC), Paris, France, 193-196, doi: 10.1109/ICNSC. 2012.6204915.

[2] Bouchery, Y., Ghaffari, A., Jemai, Z., Dallery, Y. (2012). Including sustainability criteria into inventory models, European Journal of Operational Research, Vol. 222, No. 2, 229-240, doi: 10.1016/j.ejor.2012.05.004.

[3] Chaabane, A., Ramudhin, A., Paquet, M. (2012). Design of sustainable supply chains under the emission trading scheme, International Journal of Production Economics, Vol. 135, No. 1, 37-49, doi: 10.1016/j.ijpe.2010.10.025.

[4] Benjaafar, S., Elhafsi, M. (2012). A production-inventory system with both patient and impatient demand classes, IEEE Transactions on Automation Science and Engineering, Vol. 9, No. 1, 148-159, doi: 10.1109/TASE.2011. 2158645.

[5] Zhang, B., Xu, L. (2013). Multi-item production planning with carbon cap and trade mechanism, International Journal of Production Economics, Vol. 144, No. 1, 118-127, doi: 10.1016/i.ijpe.2013.01.024.

[6] Chen, X., Benjaafar, S., Elomri, A. (2013). The carbon-constrained EOQ, Operations Research Letters, Vol. 41, No. 2, 172-179, doi: 10.1016/j.orl.2012.12.003.

[7] Ma, C.-S., Chen, X., Luo, Z.-Y., Li, T. (2015). Production strategy of considering low carbon emission policies regulation under stochastic demand, Control and Decision, Vol. 30, No. 6, 969-976, doi: 10.13195/j.kzyjc.2014.0400.

[8] Qi, Y., Tang, M., Zhang, M. (2014). Mass customization in flat organization: The mediating role of supply chain planning and corporation coordination, Journal of Applied Research and Technology, Vol. 12, No. 2, 171-181, doi: 10.1016/S1665-6423(14)72333-8. 
[9] Qu, S., Shi, X., Hua, G. (2012). Decision model for the subsides to low-carbon production by the government under the emission trading scheme, In: Zhang, Z., Zhang, R., Zhang, J. (eds.), LISS 2012 - Proceedings of $2^{\text {nd }}$ International Conference on Logistics, Informatics and Service Science, Springer-Verlag, Berlin, Germany, 57-63, doi: $10.1007 / 978-3-642-32054-510$.

[10] Mutingi, M. (2013). Developing green supply chain management strategies: A taxonomic approach, Journal of Industrial Engineering and Management, Vol. 6, No. 2, 525-546, doi: 10.3926/jiem.475.

[11] Wang, Z., Wang, C. (2015). How carbon offsetting scheme impacts the duopoly output in production and abatement: Analysis in the context of carbon cap-and-trade, Journal of Cleaner Production, Vol. 103, 715-723, doi: 10.1016/i.jclepro.2014.04.069.

[12] Sengupta, A. (2012). Investment in cleaner technology and signaling distortions in a market with green consumers, Journal of Environmental Economics and Management, Vol. 64, No. 3, 468-480, doi: 10.1016/j.jeem.2012.04. $\underline{001}$.

[13] Koren, R., Palčič, I. (2015). The impact of technical and organisational innovation concepts on product characteristics, Advances in Production Engineering \& Management, Vol. 10, No. 1, 27-39, doi: 10.14743/apem2015.1.190.

[14] Buchmeister, B., Friscic, D., Palcic, I. (2013). Impact of demand changes and supply chain's level constraints on bullwhip effect, Advances in Production Engineering \& Management, Vol. 8, No. 4, 199-208, doi: 10.14743/apem 2013.4.167.

[15] Liu, Z., Anderson, T.D., Cruz, J.M. (2012). Consumer environmental awareness and competition in two-stage supply chains, European Journal of Operational Research, Vol. 218, No. 3, 602-613, doi: 10.1016/i.ejor.2011.11. $\underline{027}$.

[16] Xu, C.-M., Zhao, D.-Z., Du, Q.-G. (2016). Decision and coordination models for supply chain with carbon emissions reduction level and price dependent demand, Control and Decision, Vol. 31, No. 3, 486-492, doi: 10.13195/j.kzyjc. $\underline{2014.1981 .}$.

[17] Li, Y.-D, Xie. X.-P, Ying, G. (2016). Research on supply chain collaboration sharing contract and decision-making mechanism under the limitation of carbon emission, Chinese Journal of Management Science, Vol. 24. No. 3, 61-70, doi: 10.16381/i.cnki.issn1003-207x.2016.03.008.

[18] Huang, Z., Zhao, D. (2014). Study on bargain model of two-echelon supply chains considering low-carbon preference of consumers, Logistics Technology, Vol. 33, No. 3, 364-368. 


\title{
Zelene proizvodne strategije za ogljično občutljive izdelke z načelom ogljične kapice
}

\author{
Ma, C. ${ }^{a}$, Liu, X..$^{\text {, }}$, Zhang, H. ${ }^{b}$, Wu, Y. ${ }^{b}$ \\ ${ }^{a}$ Tian Fu College of SouthWestern University of Finance and Economics, Chengdu, China \\ ${ }^{b}$ International Business School, Sichuan Technology and Business University, Chengdu, China
}

\begin{abstract}
P OVZETEK
V pričujočem prispevku razpravljamo o proizvodnih strategijah, ki jih uporabljajo proizvajalci ogljično občutljivih izdelkov, ki so podvrženi determinističnim in stohastičnim zahtevam. $V$ raziskavi smo preizkusili zelene proizvodne strategije za ogljično občutljive izdelke, ki se izdelujejo v skladu z ustreznimi predpisi. Predpostavili smo osnovna dva scenarija, in sicer deterministične ter stohastične zahteve. Ko politika ogljične kapice nima nobenih omejevalnih mehanizmov do proizvodnje v podjetjih se izkaže, da z naraščanjem koeficienta ogljične občutljivosti izdelka, pada tudi dobiček proizvodnih sistemov. Ko pa politika ogljične kapice (v primeru determinističnih zahtev) predstavlja za proizvajalce nekatere omejitve, je v primeru večjega koeficienta ogljične občutljivosti izdelka dobiček podjetij večji; pri stohastičnih zahtevah pa je v primeru visokega koeficienta ogljične občutljivosti izdelka dobiček podjetij nizek. Raziskava lahko služi kot vodilo za zelene proizvodne strategije pri izdelavi ogljično občutljivih izdelkov.
\end{abstract}

\section{PODATKI O ČLANKU}

Ključne besede:

Proizvodna strategija

Ogljično občutljivi izdelki

Načelo ogljične kapice

*Kontaktna oseba: uestc-vip@163.com (Liu, X.)

Zgodovina članka:

Prejet 12. junija 2016

Popravljen 5. julija 2016

Sprejet 18. julija 2016 